\title{
Polynomial chaos level points method for one-dimensional uncertain steep problems
}

\author{
P. Sochala ${ }^{\mathrm{a}, *}$, O. Le Maître ${ }^{\mathrm{b}}$ \\ ${ }^{a}$ BRGM, 3 avenue Claude Guillemin, 45060 Orléans, France \\ ${ }^{b}$ CMAP, CNRS, INRIA, École Polytechnique, Institut Polytechnique de Paris, 91128 Palaiseau, France
}

\begin{abstract}
We propose an alternative approach to the direct polynomial chaos expansion in order to approximate one-dimensional uncertain field exhibiting steep fronts. The principle of our non-intrusive approach is to decompose the level points of the quantity of interest in order to avoid the spurious oscillations encountered in the direct approach. This method is more accurate and less expensive than the direct approach since the regularity of the level points with respect to the input parameters allows achieving the convergence with low-order polynomial series. The additional computational cost induced in the post-processing phase is largely offset by the use of low-level sparse grids that require a weak number of direct model evaluations in comparison with high-level sparse grids. We apply the method to subsurface flows problem with uncertain hydraulic conductivity. Infiltration test cases having different levels of complexity are presented.
\end{abstract}

Keywords: uncertain scalar field approximation, non intrusive spectral method, preconditioning, Gibbs phenomenon, front propagation

\section{Introduction}

The goal of this work is to propose a new method to approximate the dependences of a one-dimensional scalar field on some uncertain input parameters considered random, in view of performing uncertainty analyses. The proposed approach is designed to deal with steep dependences on the uncertain parameters. The steepness, or hypersensitivity, relates here to a large variation of the solution for a small variation of the uncertain input parameters [23, 11]. Such a situation appears for instance when solving problems yielding sharp fronts with uncertain propagating velocities [20, 19]: a small change in the model parameters can change the location of the front with large changes in the solution at a given spatial location. Polynomial chaos methods $(P C)$ have been developed for uncertainty quantification and applied in many application domains [7, 9]. Classical PC methods have several advantages, such as an exponential convergence rate for smooth quantities of interest (QoI), few restrictions on the parameters distribution and moments of QoI, and direct exact derivation of the moments and sensitivity coefficients of the PC approximation. However, classical PC methods suffer from the degradation of the convergence rate when the dependences of the QoI with respect to the uncertain parameters become steep or non-smooth. Several extensions of the PC method have been proposed to improve its behavior in presence of parametric discontinuities, including the introduction of piecewise polynomial approximations [21], multiwavelet expansions [11], Padé-Legendre approximants [4], and iterated polynomial expansions [13]. More recently, preconditioning techniques using invertible transformations has been proposed as a mean to tackle the complexity of the QoI. The principle of the preconditioning is to introduce a transformation absorbing a large part of the stochastic nonlinearities of the QoI in order to improve the accuracy of the approximation. As an example, time stretching preconditioners have been developed in $[10,1,2]$ to get sparse polynomial chaos representations in uncertain dynamical systems. Nevertheless, the preconditioned PC method lacks a systematic procedure to propose appropriate transformations yielding well-conditioned approximation problems.

\footnotetext{
${ }^{*}$ Corresponding author

Email addresses: p.sochala@brgm.fr (P. Sochala), olivier.le-maitre@polytechnique.edu (O. Le Maître)
} 
In this work, we aim at proposing a more systematic approach, considering generic invertible transformations that are implicitly defined by relying on interpolation procedure based on the level points (LP) of the uncertain field. The interest of the LP method (LPM) is threefold. Firstly, it performs a preconditioning of the field to be approximated, thus avoiding the need to provide a priori an explicit transformation with prescribed structure. In addition, the transformation can be refined in an adapted fashion as detailed below. Secondly, the computational cost of constructing the approximation can be drastically reduced when the LPs defining the transformation have smooth dependences with respect to uncertain input parameters. As a result, the polynomial degree of the PC expansion of the LPs is low and can be estimated from a limited number of observations (simulations). Finally, the proposed method involves interpolation procedures that ensure the boundedness of the reconstructed approximation, contrary to the direct PCE of the field which can lead to oscillations (Gibbs phenomenon) with, for instance, violation of the maximum principle.

This paper is organized as follows. Section 2 briefly introduces the background of the PCE with the non-intrusive spectral projection method used to compute the expansion coefficients. Section 3 details the steps of the proposed method, starting with the definition of the LPs and their PC expansion, followed by the interpolation procedure to reconstruct 1D fields. Section 4 describes an adaptive version of LPs method where the approximation is refined to control the reconstruction error associated with a particular realization of the parameters. Section 5 illustrates the method on its computational efficiency on a subsurface flow problem with uncertain hydrological laws, infiltration rate, and initial saturation state. Conclusion and discussion are finally provided in Section 6.

\section{Background on polynomial chaos expansions}

Consider $u(\xi) \in \mathbb{R}$ a scalar output of a model depending on some random input parameters $\boldsymbol{\xi}=\left(\xi_{1}, \cdots, \xi_{N}\right) \in \Xi \subseteq$ $\mathbb{R}^{N}$ with probability density $p: \Xi \mapsto \mathbb{R}^{+}$. We denote $\mathbb{E}[\cdot]$ the expectation operator and $L_{2}(\Xi, p)$ the space of second order functionals, that is

$$
u(\boldsymbol{\xi}) \in L_{2}(\Xi, p) \Longleftrightarrow \mathbb{E}\left[u^{2}\right]=\int_{\Xi} u(\boldsymbol{\xi})^{2} p(\boldsymbol{\xi}) d \boldsymbol{\xi}<\infty .
$$

We shall restrict ourselves to the case of independent parameters such that $\boldsymbol{\xi}$ is a collection of independent random variables and $p$ has a product form. In the following, $\langle\cdot, \cdot\rangle$ and $\|\cdot\|_{L_{2}}$ denote the inner product of $L_{2}(\Xi, p)$ and its associated norm, respectively.

\subsection{Polynomial chaos expansion}

Let us denote $\left\{\phi_{\boldsymbol{k}}(\boldsymbol{\xi}), \boldsymbol{k} \in \mathbb{N}^{N}\right\}$ an Hilbertian basis of $L_{2}(\Xi, p)$, where $\phi_{\boldsymbol{k}}$ is a multivariate polynomial in $\boldsymbol{\xi}$ and $\boldsymbol{k}$ is a multi-index indicating the polynomial degree in the $\xi_{i}$ 's. The total degree of $\phi_{\boldsymbol{k}}$ is denoted $|\boldsymbol{k}|:=\sum_{i=1}^{N} k_{i}$. Then, $u \in L_{2}(\Xi, p)$ has the so-called Polynomial Chaos expansion

$$
u(\boldsymbol{\xi})=\sum_{\boldsymbol{k} \in \mathbb{N}^{N}} u_{\boldsymbol{k}} \phi_{\boldsymbol{k}}(\boldsymbol{\xi}), \quad u_{\boldsymbol{k}}=\left\langle u, \phi_{\boldsymbol{k}}\right\rangle=\int_{\Xi} u(\boldsymbol{\xi}) \phi_{\boldsymbol{k}}(\boldsymbol{\xi}) p(\boldsymbol{\xi}) d \boldsymbol{\xi}
$$

The PC approximation $u^{\mathcal{K}}(\boldsymbol{\xi})$ of $u(\xi)$ is obtained by truncating the expansion above to a finite series

$$
u^{\mathcal{K}}(\xi):=\sum_{k \in \mathcal{K}} u_{k} \phi_{k}(\xi)
$$

where $\mathcal{K}$ is the set of multi-indices related to the expansion. Typically, the truncation is obtained by prescribing the maximal total degree of the approximation, say $N_{\mathrm{o}}$, such that for instance

$$
\mathcal{K}\left(N_{\mathrm{o}}\right)=\left\{\boldsymbol{k} \in \mathbb{N}^{N},|\boldsymbol{k}| \leq N_{\mathrm{o}}\right\}
$$

The convergence rate of the PCE of $u$ with the truncation order $N_{\mathrm{o}}$ depends on the regularity of $u$ with respect to $\xi$ and is discussed in section 3 . 


\subsection{Non intrusive projection method}

Several methods can be used to compute the expansion and we rely in the present work on the sparse PseudoSpectral Projection [6, 5] (PSP). The LP method presented in the next section is not dependent on the specific PCprojection algorithm used to estimate the expansion coefficients, and any non-intrusive alternative can substitute. By non-intrusive, we mean an approach that uses evaluations of $u(\xi)$ at a finite number of values of $\xi$ to build the approximation in (3). The non-intrusive methods present the advantage of requiring only the availability of a deterministic solver to compute $u(\xi)$ at the requested $\boldsymbol{\xi}$.

The classical non-intrusive spectral projection uses the definition (2) and computes the expansion coefficients $u_{k}$ by evaluating $N$-dimensional integrals $[12,15]$. Typically, sparse quadrature formulas based on the partial tensorization of nested one-dimensional quadrature rules via the Smolyak's formula [17] are considered, leading to expressions of the form

$$
u_{k}=\int_{\Xi} u(\boldsymbol{\xi}) \phi_{\boldsymbol{k}}(\boldsymbol{\xi}) p(\boldsymbol{\xi}) d \boldsymbol{\xi} \simeq \sum_{j=1}^{N_{\mathrm{p}}} w_{j} u\left(\boldsymbol{\xi}_{j}\right) \phi_{\boldsymbol{k}}\left(\boldsymbol{\xi}_{j}\right),
$$

where $\left\{\boldsymbol{\xi}_{j}\right\}$ and $\left\{w_{j}\right\}$ are the $N_{\mathrm{p}}$ integration points and associated weights. The coefficients $\Pi_{\boldsymbol{k}, j}:=w_{j} \phi_{\boldsymbol{k}}\left(\boldsymbol{\xi}_{j}\right)$ define the so-called projection matrix [1] such that the vector of the expansion coefficients $\boldsymbol{u}$ is directly computed through the matrix-vector product,

$$
\boldsymbol{u}=\Pi \mathfrak{u},
$$

where $\mathbb{u}:=\left(u\left(\boldsymbol{\xi}_{1}\right), \cdots, u\left(\boldsymbol{\xi}_{N_{\mathrm{p}}}\right)\right)^{\top} \in \mathbb{R}^{N_{\mathrm{p}}}$ is the vector of the model outputs at the integration points. The PSP method differs from the classical spectral projection by using different quadrature formulas depending on the $\phi_{k}$. The quadrature formulas are selected to avoid internal aliasing, but the definition of the expansion coefficients can be eventually recast in the form of (5), albeit with a more complex definition of the projection matrix $\Pi$, see [6, 5, 22].

Finally, the PCE of $u(\xi)$ can be compactly written as

$$
u(\boldsymbol{\xi}) \approx u^{\mathcal{K}}(\boldsymbol{\xi})=\left(\Pi_{1}\right) \cdot \boldsymbol{\phi}(\boldsymbol{\xi}),
$$

where $\phi(\xi)=\left(\phi_{k}(\xi)\right)_{k \in \mathcal{K}} \in \mathbb{R}^{|\mathcal{K}|}$ is the vector basis functions. It is seen that the computational complexity for constructing the PC expansion is related to the number $N_{\mathrm{p}}$ of model evaluations (i.e. the number of quadrature points) involved in the projection. In the PSP method, this number of evaluation is directly related to the size of the set $\mathcal{K}$ retained in the expansion of $u$. The higher the truncation degree the higher the number of quadrature points needed. Further, for a given total order $N_{\mathrm{o}}, N_{\mathrm{p}}$ drastically increases with the number of parameters $N$. As a result, the convergence rate of the PC approximation is crucial for the feasibility of the projection, as high order PC expansions in high-dimension are too expensive without appropriate adaptive strategies exploiting possible sparsity in the expansion coefficients.

\section{Level Point method}

We are interested in the reconstruction of a scalar stochastic field $U(x, \boldsymbol{\xi})$ indexed by a one-dimensional physical variable $x$ in a finite interval, $x \in[a, b]$. The PC expansion introduced above can be extended by indexing the expansion coefficients on $x$. For every $x$, the convergence rate of the PC expansion depends on the smoothness of $U(x, \cdot)$ with respect to $\xi$. In the case of polynomial expansions, an exponential convergence rate with the polynomial degree is achieved for $U(x, \cdot) \in C^{\infty}(\Xi)$. We are concerned with situations where $U$ may not have such smoothness, due to localized steep dependences and gradients, possibly involving a local loss of differentiability with respect to $\xi$. These features can compromise or limit the $L_{2}$ convergence rate of the polynomial approximation which can be plagued by Gibbs phenomena, resulting in unphysical oscillations. Our main objective is to prevent such spurious oscillations in order to respect monotonicity and boundedness properties of $U$ in its approximation. To this end, we propose to construct an approximation of the stochastic level points (LPs) instead of the direct PC approximation of $U$. 


\subsection{Level Points interpolation}

For the sake of simplicity, we assume that $U(x, \xi)$ is monotonically increasing with respect to $x$ and bounded for $x \in[a, b]$. We denote $U_{-}(\xi)$ and $U_{+}(\xi)$ the values of $U$ at $x=a$ and $b$ respectively, such that it holds for almost every $\xi$ and $a \leq x_{1}<x_{2} \leq b$,

$$
U_{-}(\boldsymbol{\xi}) \leq U\left(x_{1}, \boldsymbol{\xi}\right)<U\left(x_{2}, \boldsymbol{\xi}\right) \leq U_{+}(\boldsymbol{\xi}) .
$$

The generalization of the proposed method to non-monotonic $U$ is discussed in remarks 3.1 and 3.3, as well as in the conclusion.

We start by scaling the field $U$ by means of a stochastic preconditioning $\mathcal{P}$ through

$$
Y(x, \boldsymbol{\xi}):=\mathcal{P}(U(x, \boldsymbol{\xi}))=\frac{U(x, \boldsymbol{\xi})-U_{-}(\boldsymbol{\xi})}{U_{+}(\boldsymbol{\xi})-U_{-}(\boldsymbol{\xi})} .
$$

This preconditioning maintains the monotonicity and ensures that for any $v \in[0,1]$ there is a unique $X(v, \xi) \in[a, b]$ satisfying the equation

$$
Y(X(v, \boldsymbol{\xi}), \boldsymbol{\xi})=v .
$$

We call $X(v, \cdot)$ the stochastic level point associated to level $v$. Given a finite collection $\mathbb{V}$ of $N_{1}+2>2$ ordered and distinct levels $0=v_{0}<v_{1}<\cdots<v_{N_{1}}<v_{N_{1}+1}=1$, we denote $\mathbb{X}$ the corresponding collection of level points $X_{i}(\boldsymbol{\xi})=X\left(v_{i}, \boldsymbol{\xi}\right)$ :

$$
\mathbb{X}=\left\{X_{i}(\boldsymbol{\xi}), 0 \leq i \leq N_{1}+1\right\} .
$$

Finally, for each level $v_{i} \in \mathbb{V}$, we define the stochastic scaled value $V_{i}$ from the backward transformation $\mathcal{P}^{-1}$, that is

$$
V_{i}(\boldsymbol{\xi}):=\mathcal{P}^{-1}\left(v_{i}\right)=\left(U_{+}(\boldsymbol{\xi})-U_{-}(\boldsymbol{\xi})\right) v_{i}+U_{-}(\boldsymbol{\xi}) .
$$

The final approximation of $U$, denoted $U_{\mathrm{LP}}(x, \boldsymbol{\xi})$, is obtained using a piecewise linear interpolation, namely

$$
U_{\mathrm{LP}}(x, \boldsymbol{\xi}):=\sum_{i=0}^{N_{\mathrm{l}}+1} V_{i}(\boldsymbol{\xi}) \varphi_{i}(x ; \mathbb{X})=\sum_{i=0}^{N_{1}+1}\left[\left(U_{+}(\boldsymbol{\xi})-U_{-}(\boldsymbol{\xi})\right) v_{i}+U_{-}(\boldsymbol{\xi})\right] \varphi_{i}(x ; \mathbb{X}) .
$$

In $(9)$, the $\varphi_{i}(x ; \mathbb{X})$ are the nodal functions (also called hat or $\mathbb{P}_{1}$-Lagrange functions) of the set of points (mesh) $\mathbb{X}$. These functions are piecewise linear in $x$ and such that $\varphi_{i}(x, \mathbb{X})$ is 0 for $x=X_{j \neq i}(\xi)$ and 1 for $x=X_{i}(\xi)$. Clearly, the approximation error arising from (9) comes from the interpolation error which, for sufficiently smooth functions (in $x$ ), can be controlled by selecting the number of intermediate levels $N_{1}$ and possibly the level values $v_{i}$. This is further discussed in the next section and illustrated in the numerical experiments presented later. Also, higher orderinterpolation strategies can be considered in the reconstruction formula; an interest of the piecewise linear one is the boundedness of the resulting approximation $U_{\mathrm{LP}}(x, \boldsymbol{\xi})$, which by construction has for range $\left[U_{-}(\boldsymbol{\xi}), U_{+}(\boldsymbol{\xi})\right]$. In addition, if the range of $U(x, \xi)$ is deterministic, the preconditioning step and the backward transformation are pointless and can be omitted.

Remark. The LP method can be generalized to non-monotonic functions w.r.t. $x$, if the number of LPs for all level $v_{i} \in \mathbb{V}$ remains constant for all $\boldsymbol{\xi}$. In this case, we introduce the set of LPs $Z_{i}:=\left\{X_{i, l}(\boldsymbol{\xi}), 1 \leq l \leq N_{i}^{\mathrm{z}}\right\}$, with $N_{i}^{\mathrm{Z}}$ denoting the number of LPs associated with the level value $v_{i}$, and the reconstruction formula (9) can be straightforwardly extended to accommodate this situation.

\subsection{PC-LP method}

We now proceed with the stochastic discretization of the LP method. To this end, we denote in the following $\mathbb{U}=\left\{U^{(j)}(x), j=1, \ldots, N_{\mathrm{p}}\right\}$ the PSP set containing the evaluation of $U(x, \xi)$ at the PSP nodes:

$$
U^{(j)}(x):=U\left(x, \xi_{j}\right), \quad j=1, \ldots, N_{\mathrm{p}} .
$$

The PC expansion of $U$ at the extreme points $x=a$ and $b$ is determined using the PSP method introduced in the previous section. For a given multi-index set $\mathcal{K}$, it results in PC approximations $U_{-}^{\mathcal{K}}(\xi)$ and $U_{+}^{\mathcal{K}}(\xi)$. The stochastic level points $X_{i}(\xi)$ are also approximated through a PSP procedure

$$
X_{i}(\boldsymbol{\xi}) \simeq X_{i}^{\mathcal{K}}(\boldsymbol{\xi})=\left(\Pi \mathbb{x}_{i}\right) \cdot \phi(\xi),
$$


where $\mathbb{x}_{i}:=\left(x_{i, 1}, \cdots, x_{i, N_{\mathrm{p}}}\right)^{\top}$ is the vector of the LP values associated to the level $v_{i}$ at the $N_{\mathrm{p}}$ PSP nodes $\left\{\boldsymbol{\xi}_{j}\right\}$. More precisely, $x_{i, j}$ is the unique zero of

$$
g_{i, j}(x):=U^{(j)}(x)-U^{(j)}(a)-v_{i}\left[U^{(j)}(b)-U^{(j)}(a)\right]
$$

Note that solving (11) requires in practice an interpolation procedure, since in most cases the function $U^{(j)}(x)$ will be discretized in $x$. We shall denote consistently $\mathbb{X}^{\mathcal{K}}:=\left\{X_{i}^{\mathcal{K}}(\boldsymbol{\xi}), 0 \leq i \leq N_{1}+1\right\}$ the element-wise approximation of $\mathbb{X}$, and the corresponding scaled values are approximated through

$$
V_{i}^{\mathcal{K}}(\boldsymbol{\xi})=\left(\mathcal{P}^{\mathcal{K}}\right)^{-1}\left(v_{i}\right):=\left(U_{+}^{\mathcal{K}}(\boldsymbol{\xi})-U_{-}^{\mathcal{K}}(\boldsymbol{\xi})\right) v_{i}+U_{-}^{\mathcal{K}}(\boldsymbol{\xi})
$$

Finally, the PC-LP approximation $U_{\mathrm{LP}}^{\mathcal{K}}(x, \boldsymbol{\xi})$ of $U(x, \boldsymbol{\xi})$ is

$$
U_{\mathrm{LP}}^{\mathcal{K}}(x, \boldsymbol{\xi}):=\sum_{i=0}^{N_{1}+1} V_{i}^{\mathcal{K}}(\boldsymbol{\xi}) \varphi_{i}\left(x ; \mathbb{X}^{\mathcal{K}}\right)=\sum_{i=0}^{N_{1}+1}\left[\left(U_{+}^{\mathcal{K}}(\boldsymbol{\xi})-U_{-}^{\mathcal{K}}(\boldsymbol{\xi})\right) v_{i}+U_{-}^{\mathcal{K}}(\boldsymbol{\xi})\right] \varphi_{i}\left(x ; \mathbb{X}^{\mathcal{K}}\right)
$$

To compare the proposed approach with the direct PC approximation, let us consider a discretization of $U$ over a fixed (deterministic) mesh $\Omega$ with support points $x_{0}=a<\cdots<x_{i}<\cdots<b=x_{N_{\mathrm{d}}}$; using again a piecewise linear interpolation over $\Omega$ we have

$$
U(x, \boldsymbol{\xi}) \approx \sum_{i=0}^{N_{\mathrm{d}}} U_{i}(\boldsymbol{\xi}) \varphi_{i}(x ; \Omega)
$$

Proceeding with the PC approximation of the nodal values $U_{i}(\boldsymbol{\xi})=U\left(x_{i}, \boldsymbol{\xi}\right)$ the direct approximation writes

$$
U(x, \boldsymbol{\xi}) \approx U^{\mathcal{K}}(x, \boldsymbol{\xi}):=\sum_{i=0}^{N_{\mathrm{d}}} U_{i}^{\mathcal{K}}(\boldsymbol{\xi}) \varphi_{i}(x ; \Omega) .
$$

The comparing expressions (13) and (14) highlights the essential difference between the PC-LP and the direct approximations. The latter (linearly) interpolate the PCE of $U(x, \xi)$ between fixed values of $x$, whereas the PC-LPM uses an interpolation over a $\boldsymbol{\xi}$-dependent mesh $\mathbb{X}$ of $[a, b]$, corresponding to iso-contours of the preconditioned field $Y$. Therefore, the LP method can be viewed as a preconditioned approach involving 2 successive transformations: a scaling followed by an implicit mapping from $Y(x, \cdot)$ to $X(Y, \cdot)$ which discretized at some selected values of $Y$. This mapping, schematically illustrated in Fig. 1, is invertible owing to the monotonicity in $x$ of $U(x, \cdot)$. The left plot in Fig. 1 schematizes the case of a steep variation with $x$ of $U$ at a location that depends on $\xi$. This yields a large variability of $U(x, \xi)$ as represented by the vertical extent of the gray area. The result of the LP transformation, which consists in a vertical scaling followed by a rotation of 90 degrees, is shown in the right plot of Fig. 1. It is seen that the variability of the level points $X_{i}$ at a value $Y_{i} \sim v_{i}$ is reduced, with potentially a much tighter PC spectrum requiring less computational efforts to be approximated. For instance, $U\left(x_{i}, \boldsymbol{\xi}\right)$ can be non-smooth in $\boldsymbol{\xi}$, while $X_{i}(\boldsymbol{\xi})$ can be $C^{\infty}(\Xi)$.

\subsection{Algorithms}

The PC-LP method presented above involves two steps that can be effectively distinguished: i) a pre-processing step where the approximation is constructed, and ii) a post-processing step where the approximation is queried to retrieve values of $U^{\mathcal{K}}(x, \boldsymbol{\xi})$ at points $x$ of interest and for sampled valued $\boldsymbol{\xi}(\theta)$ of the random parameters. Algorithms 1 and 2 summarize the basic computational tasks involved in the construction and sampling steps, respectively. Specifically, Algorithm 1 uses the realizations of $U$ at the nodes of the PSP method to generate the PC-LPs set $\mathbb{X}^{\mathcal{K}}$ for a prescribed number of level and the PCE of the boundary value. These outputs are used by Algorithm 2 to evaluate the corresponding PC-LP approximation at a given point $x \in[a, b]$ and a particular realization $\boldsymbol{\xi}(\theta)$ of the random input. Note that these algorithms are provided for clarity and are not designed for computational efficiency. In particular, one may want to use a less trivial evaluation algorithm if $U_{\mathrm{LP}}^{\mathcal{K}}$ is sought at multiple locations $x$ for the same realization $\boldsymbol{\xi}(\theta)$. 


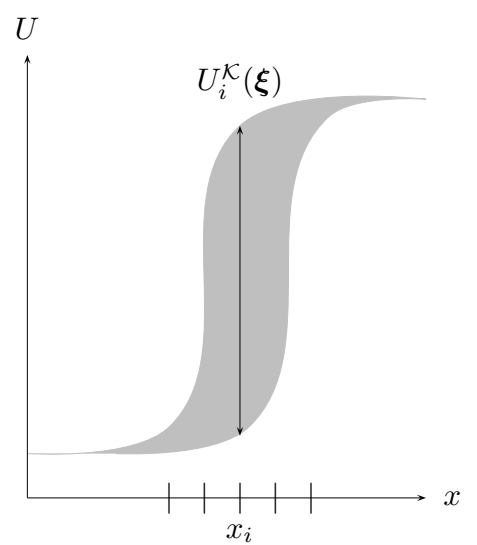

(a) Direct PC method

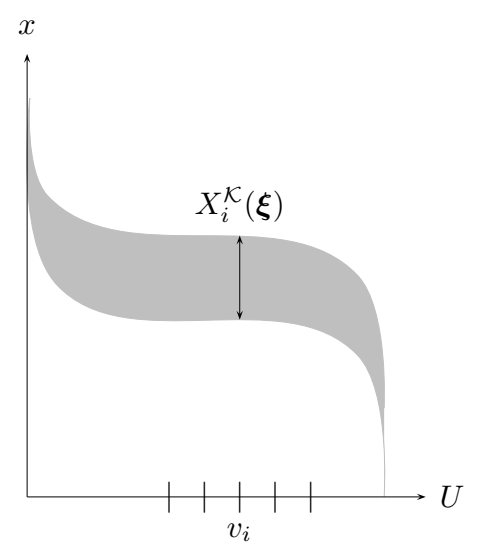

(b) PC-LP method

Figure 1: Schematical illustration of the direct PC and PC-LP methods.

Algorithm 1 shows that the PC-LP method involves some overhead to construct the approximation, in particular for finding the LPs of $U$ at the PSP nodes. In fact, as shown in the following examples, the approach is more efficient because a significantly lower number of PS nodes is needed, thanks to the smoothness of the LPs, so the computational savings results from much fewer evaluations of $U$. However, as shown in Algorithm 2, it can be anticipated that the sampling of the PC-LP approximation demands more effort than the sampling of the direct PC approximation because the interpolation mesh is changing from a realization of $\boldsymbol{\xi}$ to another. The complexity of the LP method is discussed in section 4.3.

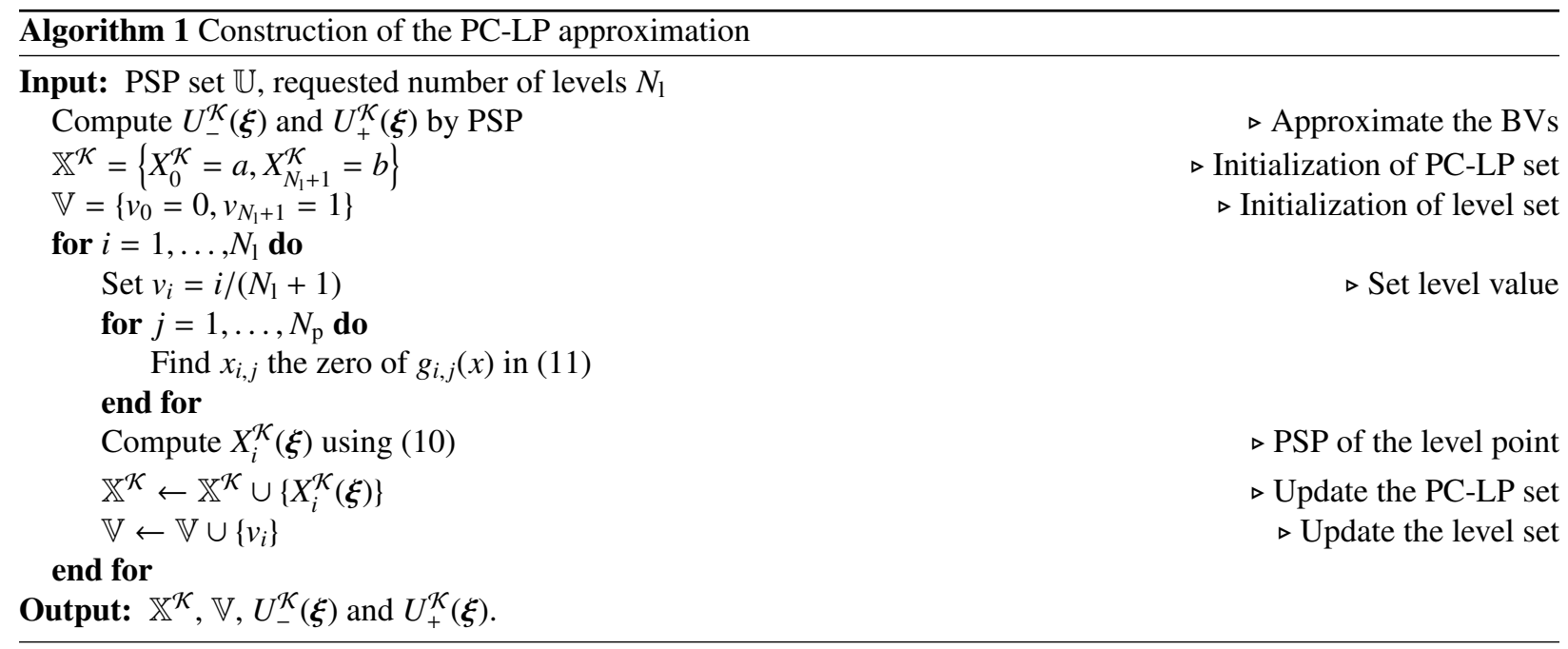

Remark. When the number of level points associated with a level $v_{i}$ is independent of $\boldsymbol{\xi}$, the generalization of the algorithm 1 to non-monotonic functions w.r.t. $x$ is possible as mentioned in Remark 3.1. The main impact of having more than one level point is that the corresponding zeros at a PSP node must be properly identified as the realization of a $X_{i}(\xi)$ or another. Similarly, the evaluation algorithm 2 can be adapted by associating a level value to each of the level points. 


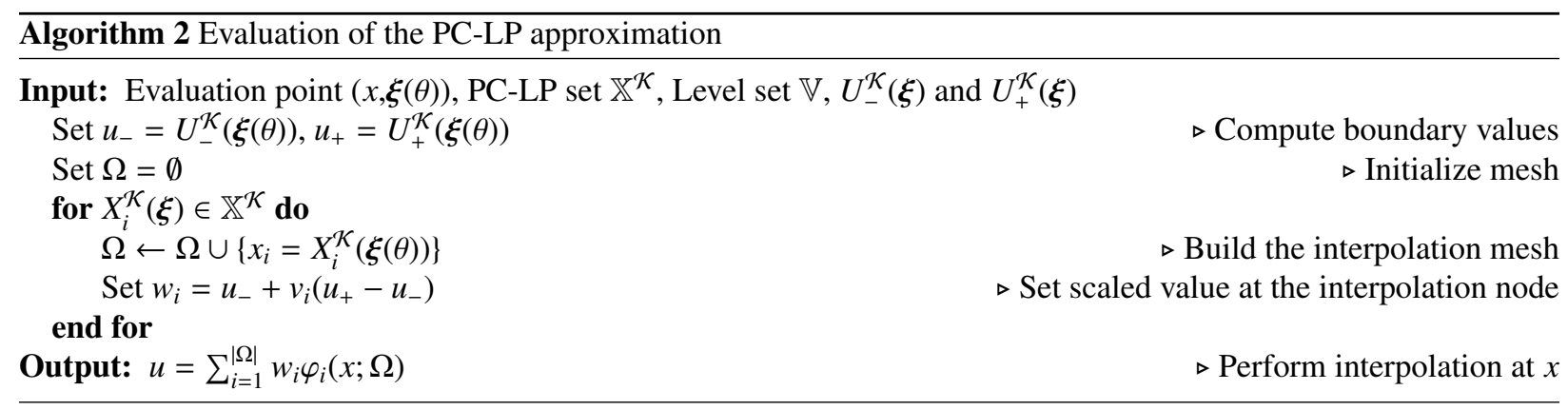

\section{Adaptive method and complexity}

As shown by (13), the PC-LP approximation consists in a piecewise linear interpolation of stochastic nodal values over a stochastic mesh consisting of the LPs in $\mathbb{X}^{\mathcal{K}}$. In fact, the expression of the scaled values $V_{i}^{\mathcal{K}}(\xi)$ in (12) indicates that the nodal values are deterministic whenever the boundary values $U_{-}$and $U_{+}$have no dependences on $\xi$. In this situation, the approximation error reduces entirely to a contribution due to the use of $\mathbb{X}^{\mathcal{K}}$ in place of $\mathbb{X}$, and contribution related to the interpolation scheme. The error on the stochastic LP can be reduced by considering more accurate estimate $X_{i}^{\mathcal{K}}(\xi)$, for instance increasing the polynomial degree of the PSP. For the latter error contribution, a mesh adaptation strategy is relevant and pursued in this section.

The benefit of the adaption can be appreciated from Fig. 2a and Fig. 2b, where a uniform and an adapted discretization of the function range have been respectively used to reconstruct a hyperbolic tangent function mimicking a front. The two discretizations use the same number $N_{1}$ of levels. For the uniform discretization of the range, the interpolation error is the largest where $U$ is mostly flat, that is in the region surrounding the domain boundaries. The error is due to the absence of interpolation points on the part of the domain with significant curvature. On the contrary, the adapted distribution of points in Fig. 2b shows a much lower level of error.

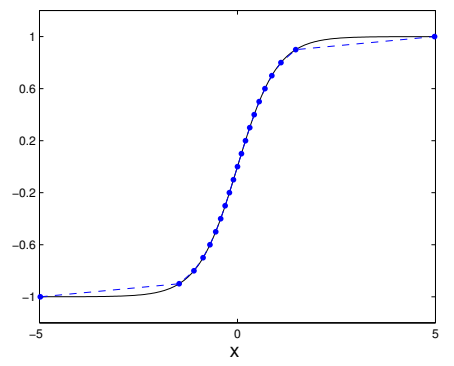

(a) Uniform discretization of the range

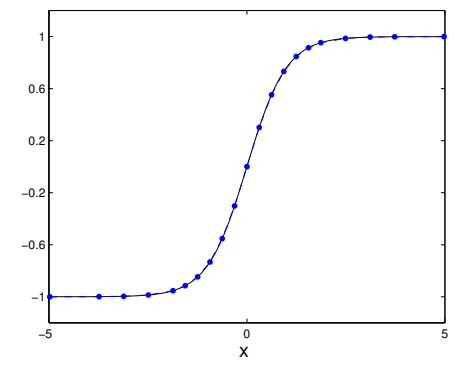

(b) Adapted discretization of the range

Figure 2: Approximation of an hyperbolic tangent function (solid line) and its piecewise linear approximation obtained with 21 points (dashed line) but different discretization of the range.

The example of the adapted discretization of the hyperbolic tangent is not complete in the sense that it concerns the approximation of a deterministic function, while in our problem $U(x, \boldsymbol{\xi})$ is random. Extending the original PCLP approximation in (13), to an adaptive approach, would consist in selecting adaptively the sequence of levels $v_{i}$ discretizing the range of $U$ in order to achieve the best interpolation error with a minimal number of levels $N_{1}$. In other words, the uniform discretization of the range would be substituted with an adapted one. The main issue with this approach is the lack of robust estimators to characterize the interpolation error (in $x$ ) in the random case, as one has to consolidate for every $x$ the error for every $\xi$. This could be achieved by sampling $\Xi$ and averaging the error estimate, but we decided to follow here an alternative route refining the PC-LP approximation for every given sampled values $\boldsymbol{\xi}(\theta)$ of the parameters. 


\subsection{Interpolation error}

The main advantage of the proposed adaptive strategy is that it works on individual samples of $U$ and therefore relies on classical error estimation. The starting point is the evaluation of the PC-LP approximation (13) for a sample value $\boldsymbol{\xi}(\theta) \in \Xi$. The sample corresponds to a deterministic mesh $\Omega$, with points $x_{i}=X_{i}^{\mathcal{K}}(\boldsymbol{\xi}(\theta))$ having associated level values $v_{i}$ and scaled values $u_{i}=V_{i}(\boldsymbol{\xi}(\theta))$. Assuming that the mesh points are indexed so $a=x_{0}<x_{1}<\cdots<x_{N_{\mathrm{l}}}<$ $x_{N l+1}=b$, we denote the mesh intervals $I_{i}:=\left[x_{i-1}, x_{i}\right]$ with lengths $h_{i}:=x_{i}-x_{i-1}$.

Considering $u \in C^{2}([a, b])$ and $\pi_{1}$ its mesh-based piecewise linear interpolation polynomial, the interpolation error on the interval $I_{i}$, defined as $e_{i}(x):=\left.\left(u(x)-\pi_{1}(x)\right)\right|_{I_{i}}$, is bounded by [14]

$$
\left|e_{i}(x)\right| \leq \frac{\left(h_{i}\right)^{2}}{8} \max _{v \in I_{i}}\left|u^{\prime \prime}(v)\right|
$$

where $u^{\prime \prime}$ is the second derivative of $u$. If $u \in C^{3}([a, b])$, a finite difference approximation of its second derivative is

$$
u_{i}^{\prime \prime} \simeq \mathcal{D}_{h}^{(2)} u_{i}:=\frac{2}{h_{i} S_{i}} u_{i-1}-\frac{2}{P_{i}} u_{i}+\frac{2}{h_{i+1} S_{i}} u_{i+1},
$$

where $S_{i}=h_{i}+h_{i+1}, P_{i}=h_{i} h_{i+1}$ and in our case $u_{i}=V_{i}(\xi(\theta))$. The computable interpolation error estimator $\mathcal{E}_{i}$ on $I_{i}$ is then defined as

$$
\mathcal{E}_{i}:=\frac{\left(h_{i}\right)^{2}}{8} \max \left(\left|\mathcal{D}_{h}^{(2)} u_{i-1}\right|,\left|\mathcal{D}_{h}^{(2)} u_{i}\right|\right)
$$

We have observed in our numerical tests that using higher order approximations of the second derivative, as a thirdorder finite difference approximation with a five-points stencil, has a weak effect on the adaptation, justifying the use of (16) to localize the interval with the maximum error.

\subsection{Adaptive algorithm}

Let us denote $l$ the index of the interval with the highest estimated error. To reduce this error, we need to introduce a new level and refine the PC-LP approximation. In the deterministic case, one would simply break $I_{l}$ into two (equal) subintervals. However, the situation is here more complex because the location of the new interpolation point cannot be explicitly specified but derives from a particular realization of a random LP defined from the prescribed level value. In other words, we have to select a level value $\left.v_{*} \in\right] v_{l-1}, v_{l}$ [ corresponding to an unknown stochastic LP $X^{\mathcal{K}}\left(v_{*}, \boldsymbol{\xi}\right)$ which should be equal to $\left(x_{l}-x_{l-1}\right) / 2$ for the realization $\xi(\theta)$; that is we have to find $v_{*}$ such that

$$
x_{*}:=X^{\mathcal{K}}\left(v_{*}, \boldsymbol{\xi}(\theta)\right)=\frac{x_{l}+x_{l-1}}{2} .
$$

We rely on a simple bisection method to solve this problem, using $v_{*}=\left(v_{l-1}+v_{l}\right) / 2$ as initial guess. The bisection process is halted when the current $x_{*}$ satisfy the criterion $\left|x_{l}+x_{l-1}-2 x_{*}\right|<2 \alpha h_{l}$ for some positive $\alpha<1$. In practice we used $\alpha=1 / 20$. The evolution of the approximation from the initial guess to the converged solution $v_{*}$ is illustrated in Fig. 3.

The most computationally intensive part of the proposed adaptive scheme is the computation and the evaluation of the stochastic LP for the successive candidates $v_{*}$. In practice, the determination of $X^{\mathcal{K}}\left(v_{*}, \boldsymbol{\xi}\right)$ amounts to finding for each PSP nodes the unique zero of the function $g_{*, j}$ defined by

$$
g_{*, j}(x)=U^{(j)}(x)-U^{(j)}(a)-v_{*}\left[U^{(j)}(b)-U^{(j)}(a)\right] .
$$

The PSP of these zeros provides the PC coefficients of $X^{\mathcal{K}}\left(v_{*}, \boldsymbol{\xi}\right)$. An important point to be underlined is the fact that the adaptation uses the same PSP set $\mathbb{U}$, and therefore requires no additional costly direct evaluation of the model. Finally, when the new level $v_{*}$ has been found, the LP set $\mathbb{X}^{\mathcal{K}}$ is completed and the indexation of the LPs is updated to maintain the ordering of the corresponding level values $v_{i}$. The procedure can then repeated to yield the next level and reduce further the interpolation error. Algorithm 3 outlines the adaptive procedure for a given realization $\boldsymbol{\xi}(\theta)$. The algorithm has two stopping criteria: one based on the maximum number of levels and a second one which halts the adaptation when the interpolation error bound becomes lower than an a priori tolerance $\epsilon_{\mathrm{tol}}$. The output of the algorithm is an adapted set $\mathbb{X}^{\mathcal{K}}$ of stochastic level points and corresponding level values $\mathbb{V}=\left\{v_{i}\right\}$. 


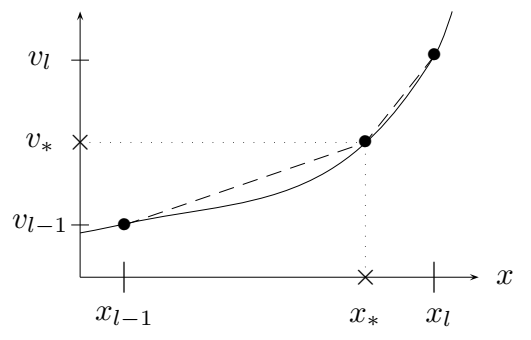

(a) Initialization

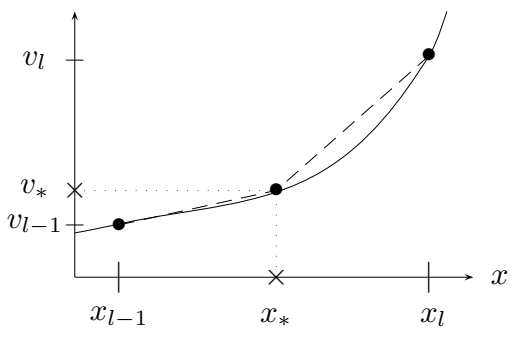

(b) Convergence

Figure 3: New interpolation point $x_{*}$ in the interval $I_{l}$, at initialization using $v_{*}=\left(v_{l-1}-v_{l}\right) / 2$ and at convergence. Note the effect on the interpolation error.

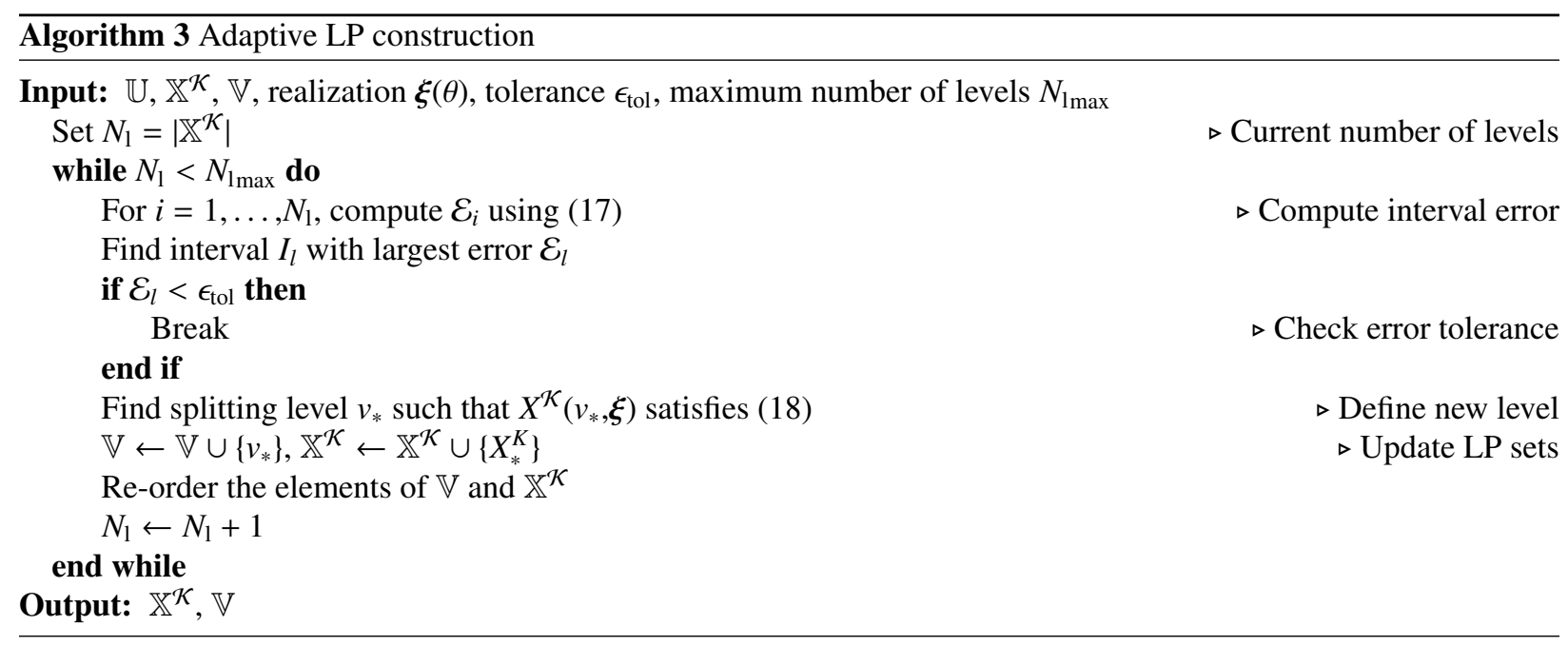

Whence Algorithm 3 has been applied, the evaluation of $U^{\mathcal{K}}(x, \xi(\theta))$ can be performed using Algorithm 2 for any value of $x \in[a, b]$. Evaluation of $U$ at other realizations of $\xi$ is also possible using Algorithm 2, although the approximation may not be adapted with possibly larger interpolation error. In fact, Algorithm 3 can be employed to generate the sets of levels and LPs, $\mathbb{V}$ and $\mathbb{X}^{\mathcal{K}}$, yielding an interpolation error less than $\epsilon_{\mathrm{tol}}$ with controlled probability. This goal can be achieved through successive calls of Algorithm 3 for (randomly) sampled values of $\boldsymbol{\xi}$, possibly by allowing progressively an increase of the size of $\mathbb{V}$. However, it is important to stress that the proposed approach reduces the interpolation error of the reconstruction, which is only one the error contributions. Consequently, the error $U-U_{\mathrm{LP}}^{\mathcal{K}}$ does not vanish when $\epsilon_{\mathrm{tol}} \rightarrow 0$, and the tolerance should be consistent with the PSP error, and possibly with the discretization error in the elements of $\mathbb{U}$. Balancing these errors is an important topic that will be investigated in the future. In particular, in the case of very fine discretizations of the range, it could be that (18) has no solution $v_{*} \in\left[v_{l-1}, v_{l}\right]$ because of the finite PC discretization, though this situation never happen in our numerical tests, even for the lowest PC orders, owing to the fast convergence the LPs approximations.

\subsection{Complexity}

We complete this section by a brief discussion on the computational complexity, assuming that the PC-LP method will be used to estimate statistics of $U$ from $M$ samples $\xi(\theta)$. The CPU time to generate $M$ samples of the PC-LP approximation is separated into the construction and the evaluation times.

The construction time $T_{c}$ can be estimated by

$$
T_{c} \propto \overline{T_{1}} N_{\mathrm{p}}+T_{2} N_{\mathrm{p}} N_{\mathrm{l}} C_{\text {adapt }},
$$


where the first term $\overline{T_{1}} N_{\mathrm{p}}$ accounts for the deterministic model evaluations $U^{(j)}$ at the $N_{\mathrm{p}}$ PSP nodes, $\overline{T_{1}}$ being the PSP node-averaged time as this time may vary from a node to another. The second term is the time to compute $\mathbb{X}, \mathbb{V}$ as well as $U_{-}$and $U_{+}$; it is essentially proportional to the number of levels $N_{1}$ and the number of PSP nodes $N_{\mathrm{p}}$, with a time $T_{2}$ corresponding to the (averaged) time for finding the zero of a $g_{i, j}$. Note that the computational time for applying the PSP operator, whence all nodal values are known, is negligible in our numerical experiments. Finally, the constant $C_{\text {adapt }}$ depends on the adaptive strategy used. Its value can range from $C_{\text {adapt }}=1$ in the case of a non-adapted PC-LP method with $N_{1}$ pre-selected levels, to $C_{\text {adapt }}=M$ in the case where $N_{1}$ adapted levels are determined for every of the $M$ samples.

The evaluation time $T_{e}$ can be estimated by

$$
T_{e} \propto T_{3}(\mathcal{K}) N_{1} M+T_{4}\left(N_{1}\right) M .
$$

The first contribution to $T_{e}$ reflects the evaluation of the PC expansions for the $N_{1}$ stochastic LPs and boundary values (see Algorithm 2) and for the $M$ samples, and therefore depends on the PC basis defined by $\mathcal{K}$. In fact, because the basis functions must be evaluated only once per sample, the constant $T_{3}(\mathcal{K})$ is essentially proportional to the basis dimension $|\mathcal{K}|$, reflecting the complexity of the dot product between basis functions and PC coefficients. Finally, the last contribution $T_{4}\left(N_{1}\right) M$ measures the interpolation time necessary to evaluate (13) at the set of the $x$ requested.

\section{Results}

We illustrate the PC-LP method on a 1D subsurface flow problem with uncertain hydrological law. Specifically, we focus on the prediction of the water pressure field which is the QoI in various applications such as the water resources forecast, the study of infiltration processes, and the hydrological parameter inference.

\subsection{Physical model and deterministic solver}

We consider subsurface flows described by the 1D Richards' equation [16] in conservative form,

$$
\partial_{t}(\theta(\psi))-\partial_{z}\left(K(\psi)\left(\partial_{z} \psi-1\right)\right)=0
$$

which combines the water mass conservation principle and the Darcy's law. In (22), $z$ is the (downward oriented) vertical coordinate, $\psi(z, t)$ the pressure head, $\theta(\psi)$ the volumetric water content, and $K(\psi)$ the hydraulic conductivity. The nonlinear parabolic equation (22) is equipped with an initial condition $\psi(\cdot, 0)=\psi^{0}$ and Dirichlet or Neumann boundary conditions at the top $(z=0)$ and bottom $(z=L)$ boundaries of the domain. We shall use $\psi(A, \cdot)=\psi_{D}^{A}$ or $v(A, \cdot)=v_{N}^{A}$ for $A \in\{0, L\}$, where $v(\psi)=-K(\psi)\left(\partial_{z} \psi-1\right)$ is the flow rate, and $\psi_{D}^{A}$ and $v_{N}^{A}$ denote the Dirichlet and the Neumann data prescribed at $x=A$. The hydrological laws are described by the Brooks-Corey's relations [3],

$$
\theta(\psi)=\left\{\begin{array}{ll}
\theta_{\mathrm{s}}\left(\psi / \psi_{\mathrm{a}}\right)^{-\frac{1}{b}} & \text { if } \psi<\psi_{\mathrm{a}}, \\
\theta_{\mathrm{s}} & \text { if } \psi \geq \psi_{\mathrm{a}},
\end{array} \quad K(\psi)= \begin{cases}K_{\mathrm{s}}\left(\psi / \psi_{\mathrm{a}}\right)^{-\gamma} & \text { if } \psi<\psi_{\mathrm{a}}, \\
K_{\mathrm{s}} & \text { if } \psi \geq \psi_{\mathrm{a}},\end{cases}\right.
$$

where $\theta_{\mathrm{s}}$ is the saturated water content, $K_{\mathrm{s}}$ the saturated hydraulic conductivity, $\psi_{\mathrm{a}}$ the air entry pressure head, $b$ an exponent related to the pore-size distribution and $\gamma:=2+3 b^{-1}$. In the following, we use $\theta_{\mathrm{s}}=0.562, \psi_{\mathrm{a}}=-4.55 \mathrm{~cm}$, $b=13.3$, while $K_{\mathrm{S}}$ is assumed to be uncertain but the set of the hydrological parameters can be treated as uncertain as we have done in [18]. The stochastic parametrization of $K_{\mathrm{S}}$ is discussed in the examples below.

The QoI is the pressure field at a final time $T>0$, and $\psi(z, T)$ is simply denoted $\psi(z)$ hereafter. The physical model is solved with a Discontinuous Galerkin (DG) method on a uniform spatial mesh, a backward differentiation formula for the time discretization and a Newton linearization of the non-linear terms (see [18] for more details). Preliminary tests have been performed to select the mesh size $h$ and a time step $\delta t$ ensuring sufficiently converged deterministic approximations over the whole uncertain parameters range, and these discretization parameters are kept fixed in all subsequent simulations. For the PC-LP approximation of the uncertain pressure, a continuous affine reconstruction interpolating the DG solution at the center of the spatial elements is used for the PSP realizations $U^{(j)}$. 


\subsection{Case of a single uncertain parameter}

\subsubsection{Problem description}

For illustration and validation purposes, we first consider the case of an infiltration in an homogeneous soil with one uncertain parameter, the constant saturated conductivity $K_{\mathrm{s}}$. We set $L=50 \mathrm{~cm}, T=30 \mathrm{~min}, \psi_{D}^{0}=0, \psi_{D}^{L}=\psi^{0}=$ $-1 \mathrm{~m}$, while $K_{\mathrm{s}}\left[\mathrm{cm} \cdot \mathrm{s}^{-1}\right]$ is modeled as a random variable with log-uniform distribution over the decade $\left[10^{-4}, 10^{-3}\right]$. The parametrization of $K_{\mathrm{s}}$ uses a single canonical random variable $\xi$ with uniform distribution in the unit interval:

$$
K_{\mathrm{s}}(\xi)=10^{\xi-4}, \quad \xi \sim \mathcal{U}[0,1] .
$$

Fig. 4 shows the computed pressure head field at time $T$ for the extreme and mean values of $K_{\mathrm{s}}$. The pressure field is monotonic w.r.t. $z$ and exhibits a steep wetting fronts whose location varies with $K_{\mathrm{s}}$ because its velocity is increasing with saturated conductivity. As a result, the variability of the pressure head is larger in the right part of the domain where the front can be found and is zero in areas that are not crossed by the front at the considered time.

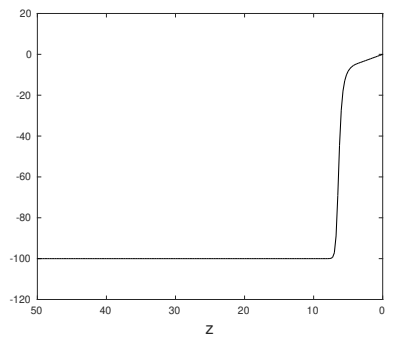

(a) $K_{\mathrm{s}}=10^{-4}$

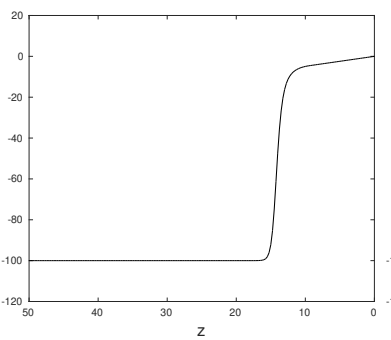

(b) $K_{\mathrm{s}}=3.91 \times 10^{-4}$

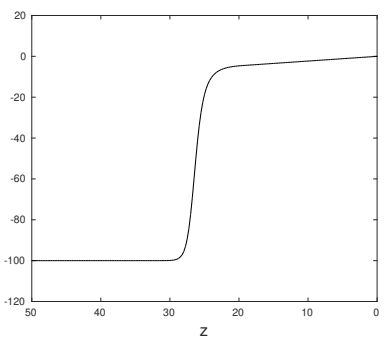

(c) $K_{\mathrm{s}}=10^{-3}$

Figure 4: Pressure head for the extreme (left and right) and mean (center) values of $K_{\mathrm{s}}$. Problem of Section 5.2.1.

\subsubsection{PC-LP approximations}

The direct PCE (14) is first performed using an increasing PC order $N_{\mathrm{o}}=10,20$, and 50. An evaluation of these direct PC expansions is shown in Fig. 5 for the mean $K_{\mathrm{s}}$. The impact of the steep variation in the pressure head at a location that depends on $\xi$ is clearly noticeable for $z \in[10,30]$, with a pollution of the approximation by large spurious oscillations. These oscillations are also present in the reconstruction at other values of $K_{\mathrm{S}}$ (not shown). As the pressure head field is smooth in $z$, the oscillations decay slowly with the polynomial order and eventually become barely visible for $N_{\mathrm{o}}=50$. Such large PC order would call for too many PSP nodes for the evaluation of the PC coefficients in higher dimension, thus compromising the applicability of the direct approach to problems with more than few uncertain parameters.

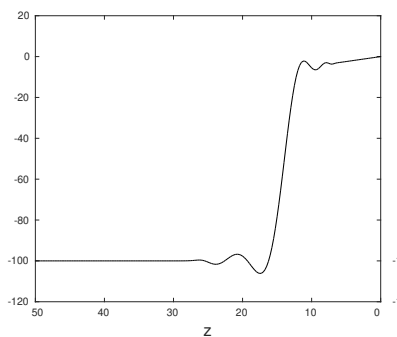

(a) $N_{\mathrm{o}}=10$

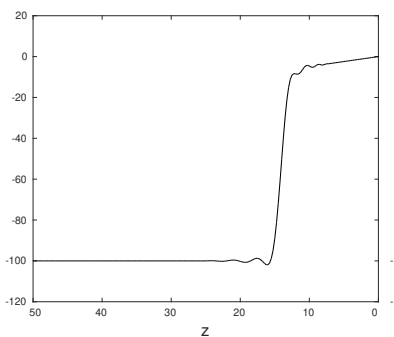

(b) $N_{\mathrm{o}}=20$

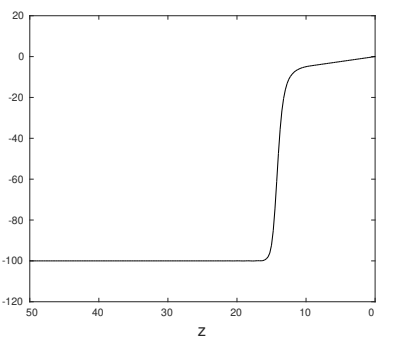

(c) $N_{\mathrm{o}}=50$

Figure 5: Evaluations of the direct PC approximation of the pressure head for the mean $K_{\mathrm{S}}$ at orders $N_{\mathrm{o}}=10,20,50$ as indicated. Problem of Section 5.2.1.

In contrast to the direct PC method, Fig. 6 shows the approximation of the pressure head obtained with the PC-LP method using $N_{\mathrm{o}}=4$ only. The plots in Fig. 6 show the PC-LP reconstruction for the value of $\xi$ corresponding to the mean value of $K_{\mathrm{S}}$ and using a uniform discretization of the pressure head range with $N_{\mathrm{l}}=10$ (left) and $N_{\mathrm{l}}=20$ 
(right) levels. It is seen that even though the polynomial degree used, $N_{\mathrm{o}}=4$, is much less than for the direct PC method shown above, no spurious oscillations are polluting the approximation. In addition, the maximum principle is satisfied.

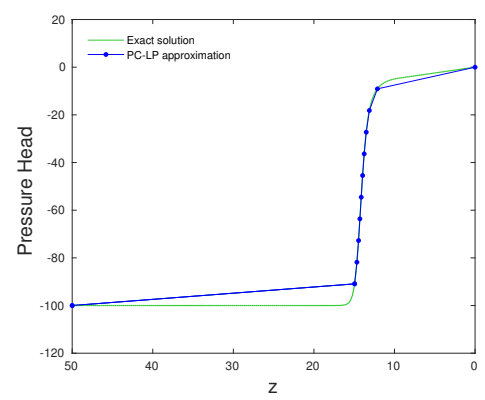

(a) $N_{\mathrm{l}}=10$

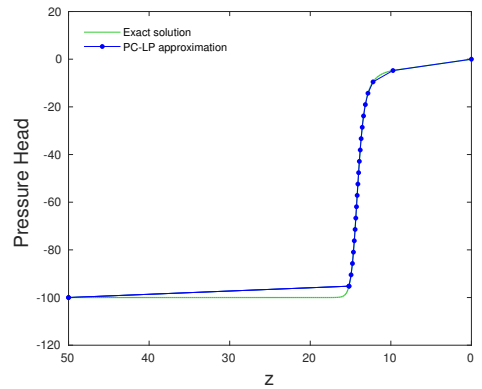

(b) $N_{\mathrm{l}}=20$

Figure 6: Exact solution (computed from DG deterministic solver) for the mean $K_{\mathrm{s}}$ (solid line) and its uniform PC-LP approximation (bullets) using $N_{\mathrm{o}}=4$. The number of level $N_{\mathrm{l}}$ in the approximations is indicated. Problem of Section 5.2.1.

The plots in Fig. 6 highlights the possibly large error and slow convergence of the PC-LP method when a uniform discretization of the range is used. In particular, the linear reconstruction from the level points will induce an error in $O\left(1 / N_{1}\right)$ over a large portion of the domain (here for $z>15$ ). This error is efficiently reduced by using the adaptive method, as illustrated in Fig. 7, where the adaptive PC-LP approximation is reported at several iterations of Algorithm 3 and for the same value of $K_{\mathrm{s}}$. Fig. 7 highlights the improvement of the convergence arising from the adaptivity, as the approximation with 16 LPs (right plot) is indistinguishable from the exact solution when the non-adapted approach with 20 LPs has a significant error (see Fig. 6b).

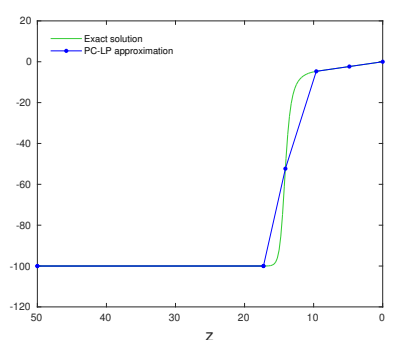

(a) Iteration $3-N_{1}=4$

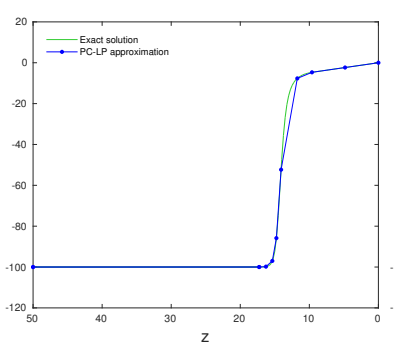

(b) Iteration $7-N_{1}=8$

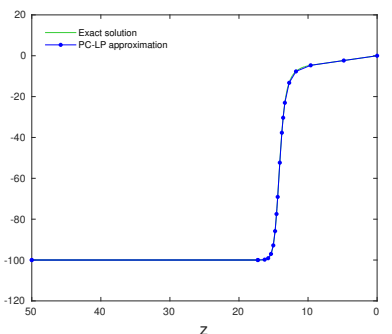

(c) Iteration $15-N_{1}=16$

Figure 7: Exact solution (computed from DG deterministic solver) for the mean $K_{\mathrm{S}}$ (solid line) and its adaptive PC-LP approximation (bullets) using $N_{\mathrm{o}}=4$. The number of level $N_{\mathrm{l}}$ in the approximations is indicated. Problem of Section 5.2.1.

\subsubsection{Error analysis}

The error of the different PC-LPs approximations is measured using a Monte Carlo (MC) estimate of the mean squared error, say $\widehat{e}(z)$, defined by

$$
\widehat{e}(z):=\frac{1}{M} \sum_{i=1}^{M}\left[\psi_{h}\left(z, \xi\left(\theta_{i}\right)\right)-\psi_{\mathrm{LP}}^{\mathcal{K}}\left(z, \xi\left(\theta_{i}\right)\right)\right]^{2},
$$

where $\xi\left(\theta_{i}\right)$ are the MC samples drawn from the density $p(\xi)$. In practice, $M=10^{3}$ are used in the estimation of $\widehat{e}(z)$.

Fig. 8 presents the error $\widehat{e}(z)$ for the uniform (left) and adapted (right) level discretizations with $N_{1}=30$, and using PC approximation with $N_{\mathrm{o}}=2,3$ and 4 as indicated. For $N_{\mathrm{o}}=2$ the errors of the uniform and adapted approximations are comparable for $z \in[0,30]$, while for $z>30$ the error of the adapted approach is much less than for the uniform one. Also, when increasing the PC order, the error is seen to remain essentially constant in the lower part of the 
column $(z>30)$. This suggests that in this part the error is dominated by the level discretization and not by the PC approximation, as one would have expected from the analysis of the solutions for the mean $K_{\mathrm{s}}$ reported above. For the upper part of the column, $z<30$, the uniform and adapted methods show a different behavior with the order $N_{\mathrm{o}}$. While the error of the adapted PC-LP method continues to decay when $N_{\mathrm{o}}$ increases, the error of the uniform method stagnate after $N_{\mathrm{o}}=3$. Again, a dominant level discretization error can be blamed in the uniform case, when the adaptation with only $N_{1}=30$ levels is seen to yield an error dominated by the PC approximations up to $N_{\mathrm{o}}=4$.

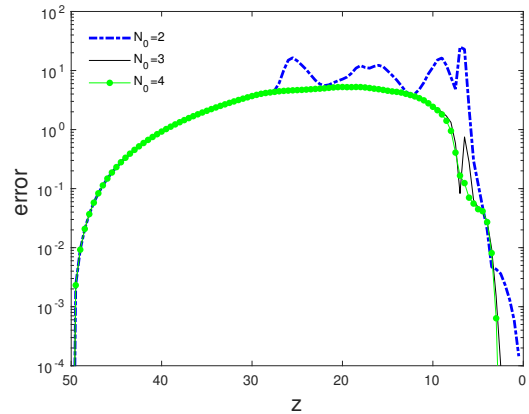

(a) Uniform discretization

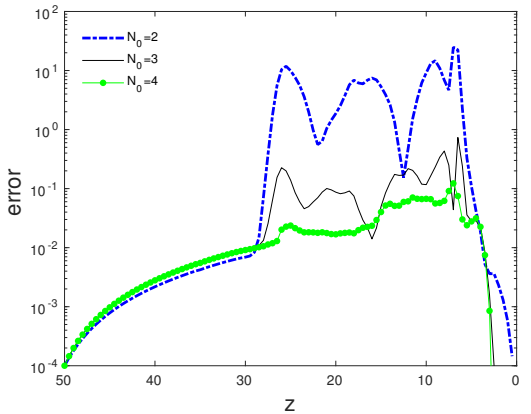

(b) Adapted discretization

Figure 8: Error $\widehat{e}(z)$ for the uniform and adapted level selection and $N_{\mathrm{l}}=30$. The errors are reported for $N_{\mathrm{o}}=2,3$ and 4. Problem of Section 5.2.1.

Fig. 9 shows the errors of the PC-LP method for the uniform and adapted level selection, in the case of $N_{\mathrm{o}}=4$. The errors are reported for $N_{1}=20,30$ and 40. The curves show that the error decays when more levels are involved in the construction, and with a faster rate and lower values in the adaptive approach. The decay of the error with $N_{1}$ confirms that the PCE approximation error is here small; however, it is expected that the error will completely level-off for larger $N_{1}$.

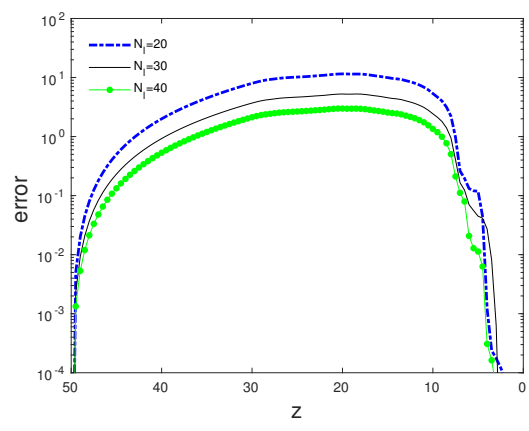

(a) Uniform discretization

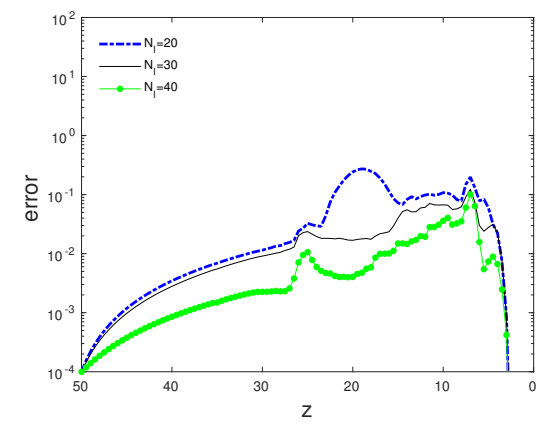

(b) Adapted discretization

Figure 9: Error $\widehat{e}(z)$ for the uniform and adapted level selection and $N_{\mathrm{o}}=4$. The errors are reported for $N_{\mathrm{l}}=20,30$ and 40 . Problem of Section 5.2.1.

The behavior of the PC-LP error can also be appreciated from Table 1, which reports the MC estimates of the space-integrated mean-squared errors (23) using their approximated value at the centers $z_{i}$ of the spatial mesh elements,

$$
\widehat{E}:=\sqrt{h \sum_{i=1}^{N_{\mathrm{x}}} \widehat{e}\left(z_{i}\right)^{2}},
$$

where $N_{\mathrm{x}}=100$ is the number of elements and $h=0.5 \mathrm{~cm}$. In particular, the results stress the importance of increasing jointly $N_{\mathrm{o}}$ and $N_{1}$ to effectively reduce the error, as well as the improvements brought by the adaptivity. 


\begin{tabular}{|c|ccc|ccc|}
\hline & \multicolumn{3}{|c|}{ Uniform discretization } & \multicolumn{3}{c|}{ Adapted discretization } \\
$N_{\mathrm{o}}$ & $N_{\mathrm{l}}=20$ & $N_{\mathrm{l}}=30$ & $N_{\mathrm{l}}=40$ & $N_{\mathrm{l}}=20$ & $N_{\mathrm{l}}=30$ & $N_{\mathrm{l}}=40$ \\
\hline 3 & $4.80 \times 10^{1}$ & $2.21 \times 10^{1}$ & $1.27 \times 10^{1}$ & 1.13 & $8.77 \times 10^{-1}$ & $8.08 \times 10^{-1}$ \\
4 & $4.77 \times 10^{1}$ & $2.18 \times 10^{1}$ & $1.24 \times 10^{1}$ & $6.37 \times 10^{-1}$ & $2.19 \times 10^{-1}$ & $1.23 \times 10^{-1}$ \\
5 & $4.77 \times 10^{1}$ & $2.18 \times 10^{1}$ & $1.24 \times 10^{1}$ & $5.93 \times 10^{-1}$ & $1.94 \times 10^{-1}$ & $1.03 \times 10^{-1}$ \\
\hline
\end{tabular}

Table 1: Integrated error $\widehat{E}$ for PC order $N_{\mathrm{o}}=3,4$ and 5 and number of levels $N_{\mathrm{l}}=20,30$ and 40. Problem of Section 5.2.1.

To better understand the effect of adaptivity, we provide in Fig. 10 plots of approximation $\psi_{\mathrm{LP}}^{\mathcal{K}}(\xi)$ versus the "exact" value $\psi_{h}(\xi)$, for the values of $\xi$ in the MC set, and the uniform and adapted methods. These so-called Q-Q plots are considered at locations $z=10 \mathrm{~cm}$ and $z=27 \mathrm{~cm}$. At $z=10 \mathrm{~cm}$, the uniform and adaptive PC-LP methods yield comparable errors, while the improvement brought by the adaptivity is very significant at $z=27 \mathrm{~cm}$. Indeed, at this location that is reached by the front only for the highest values of $K_{\mathrm{s}}$, the uniform approach yields a large error for all events corresponding to a front remaining upstream. On the contrary, the adapted approach is able to control the error of all events.

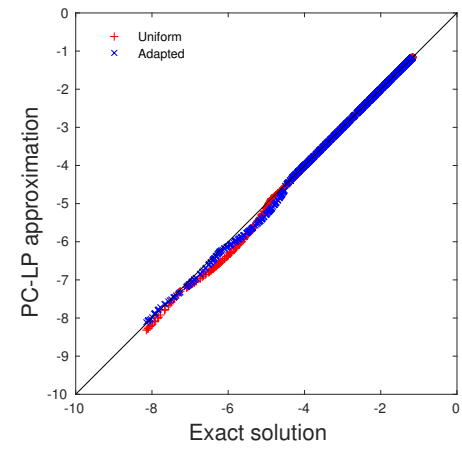

(a) $z=10 \mathrm{~cm}$

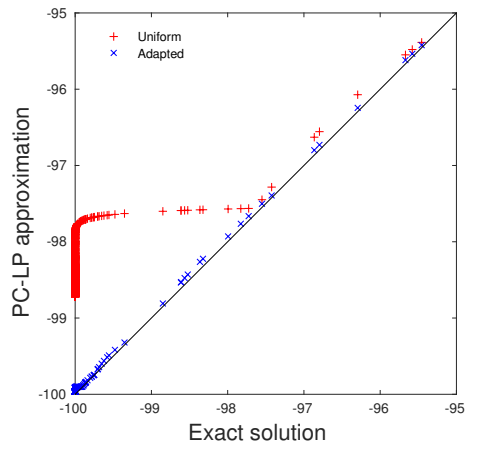

(b) $z=27.5 \mathrm{~cm}$

Figure 10: PC-LP approximations versus exact solution at two selected spatial locations. Numerical parameters are $N_{\mathrm{o}}=5$ and $N_{\mathrm{l}}=40$. Problem of Section 5.2.1.

\subsection{Higher dimensional problem}

\subsubsection{Homogeneous $K_{\mathrm{s}}$}

The objective of this problem is to test and illustrate the PC-LP method on a situation involving a higher number of uncertain input parameters. To this end, we fix $L=40 \mathrm{~cm}$ and $T=2 \mathrm{~h}$ and investigate the effects of uncertainties in the boundary and initial conditions, and in the saturated conductivity. For the boundary condition, we consider a random Neumann datum $v_{\mathrm{N}}^{0}\left[\mathrm{~mm} \cdot \mathrm{h}^{-1}\right]$ at the top of the soil column, assuming a uniform distribution in the range $[2,6]$ (moderate rainfall intensity). Using the canonical random variable $\xi_{1}$ to parameterize $v_{\mathrm{N}}^{0}$, it comes

$$
v_{\mathrm{N}}^{0}\left(\xi_{1}\right)=2+4 \xi_{1}, \quad \xi_{1} \sim \mathcal{U}[0,1]
$$

For the initial condition, we assume an uncertain initial saturation state $\psi^{0}[\mathrm{~cm}]$ in the range $[-120,-80]$ with, again, a uniform distribution; to enforce the independence between the uncertain boundary and initial condition, we use a second canonical random variable $\xi_{2}$ and write the initial condition as

$$
\psi^{0}\left(\xi_{2}\right)=-120+40 \xi_{2}, \quad \xi_{2} \sim \mathcal{U}[0,1]
$$

The remaining boundary condition at the bottom of the domain is held equal to the initial condition, $\psi_{D}^{L}\left(\xi_{2}\right)=$ $\psi^{0}\left(\xi_{2}\right)$. As in the previous example saturated conductivity field $K_{\mathrm{s}}$ is also uncertain with $\log$-uniform distribution 
and parametrized with a third independent canonical random variable $\xi_{3}$ through

$$
K_{\mathrm{s}}\left(\xi_{3}\right)=10^{\xi_{3}-4}, \quad \xi_{3} \sim \mathcal{U}[0,1] .
$$

The problem then involves independent uncertainty sources, whose individual effects can be appreciated from Fig. 11, where the one-at-a-time analyses about the median value of the parameters (i.e. $\left.\xi_{i}=1 / 2\right)$ are reported. It is seen that in terms of individual effects the (upper) boundary condition mainly affects the location of the front, the initial condition changes the solution in the lower part of the domain where it is spatially constant, and the saturated conductivity principally changes the steepness of the front.

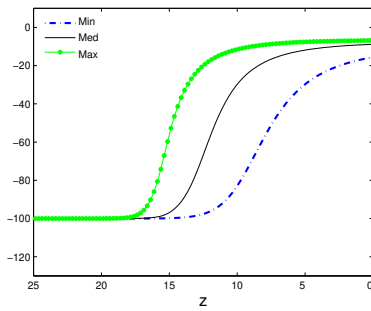

(a) Boundary Condition

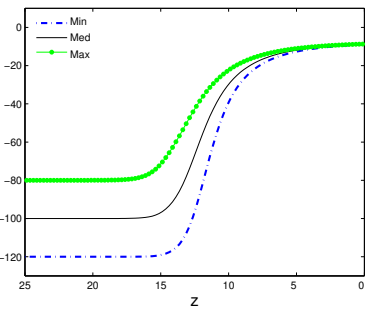

(b) Initial Condition

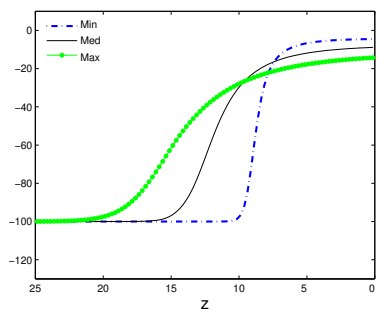

(c) Saturated conductivity

Figure 11: One-at-a-time influence of uncertain boundary condition, initial condition and saturated conductivity field. Plotted are the solution for the minimal, median and maximal values in the respective range of variation. Problem of Section 5.3.1.

For the PC-LP, we use the PSP method at levels $\ell=2, \ldots, 4$ corresponding to a number of (Féjèr) nodes $N_{\mathrm{p}}=31$, 111 and 351. From Fig. 11 we observe the structure of the solution, which is monotonically increasing w.r.t. $z$ with two plateaus at the two ends of the domain. This suggests an a priori discretization in levels that concentrate around to 0 and 1 to obtain more evenly distributed level points in space, with lower interpolation error as a result. Following this idea, we consider the $N_{1}$ Chebyshev points of the unit interval,

$$
v_{l}:=\left[1-\cos \left(l \pi /\left(N_{1}+1\right)\right)\right] / 2, \forall l=1 \cdots N_{1} .
$$

The Chebyshev discretization is contrasted with the case of the uniform discretization of $[0,1]$ on the resulting error. The errors $\widehat{e}(z)$ given by (23) are reported in Fig. 11 for $N_{1}=30$ intermediate levels. The plots confirm the improvement on the error brought by the non-uniform discretization, in particular at the bottom of the domain where the solution is spatially constant. In contrast, we observe marginal improvements of the error when the level $\ell$ of the PSP method increases. This finding suggests that the PC discretization error is dominated by the level discretization. This is further confirmed by Fig. 13, which reports the errors for several numbers of level $N_{1}$ and a fixed PSP-level $\ell=3$. It is seen that the error decreases for both the uniform and non-uniform discretizations of the range when $N_{1}$ goes from 20 to 40 .

\subsubsection{Non-homogeneous $K_{\mathrm{s}}$}

To complete the tests on the PC-LP method, we now turn to the case of an uncertain and non-homogeneous saturated conductivity field $K_{\mathrm{s}}$. The field is assumed to be stationary with a log-normal distribution, that is $y(z, \theta):=$ $\log \left(K_{\mathrm{s}}(z, \theta)\right)$ is a Gaussian stochastic process. The process $y$ can be decomposed into the Karhunen-Loève expansion (KLE) [8],

$$
y(z, \theta)=\mu_{y}+\sum_{i=1}^{\infty} \sqrt{\lambda_{i}} y_{i}(z) \eta_{i}(\theta),
$$

where $\mu_{y}$ is the mean of $y$, the couples $\left\{\left(\lambda_{i}, y_{i}(z)\right)\right\}$ are the eigenpairs of the covariance function $\mathcal{K}\left(z, z^{\prime}\right)$ of $y$, and the $\xi_{i}(\theta)$ are uncorrelated standard Gaussian random variables. In practice, we rely on a piecewise linear numerical approximation of the eigenfunction, consistently with the discretization of the pressure field, and a weak formulation of the homogeneous Fredholm integral equation of the second kind satisfied by $y_{i}$. In this work we use the squareexponential covariance function

$$
\mathcal{K}\left(z, z^{\prime}\right):=\sigma_{y}^{2} \exp \left[-\left(z-z^{\prime}\right)^{2} /\left(2 l_{\mathrm{c}}^{2}\right)\right],
$$




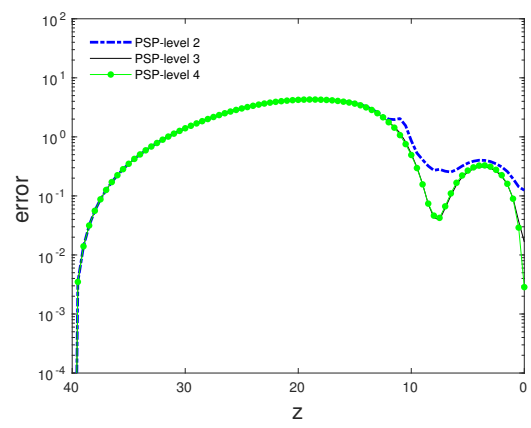

(a) Uniform discretization

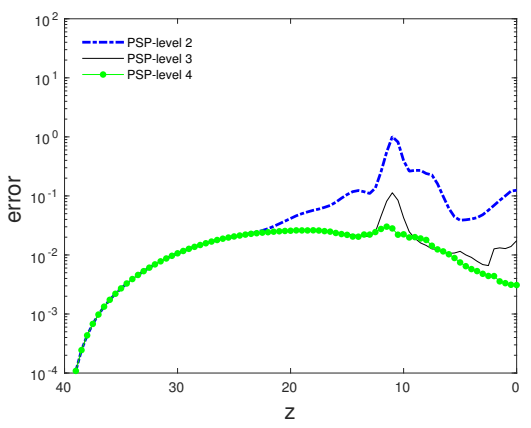

(b) Chebyshev discretization

Figure 12: Error $\widehat{e}(z)$ for the uniform and Chebyshev discretization of the range and $N_{1}=30$. The errors are reported for different PSP levels $\ell$. Problem of Section 5.3.1

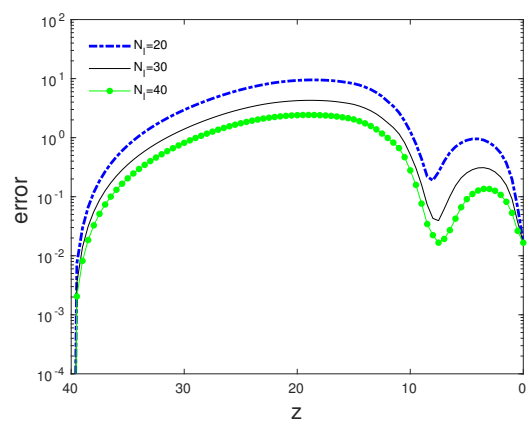

(a) Uniform discretization

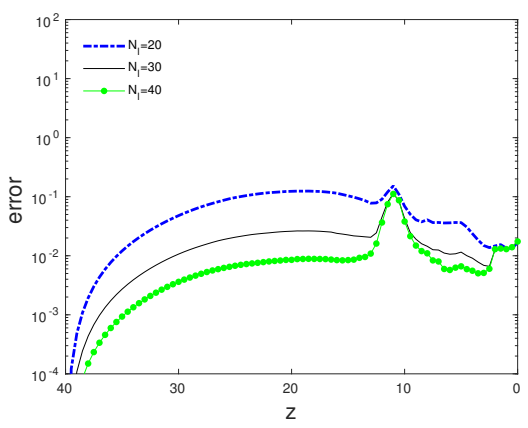

(b) Chebyshev discretization

Figure 13: Error $\widehat{e}(z)$ for the uniform and Chebyshev discretization of the range and $\ell=3$. The errors are reported for $N_{\mathrm{l}}=20,30$ and 40 . Problem of Section 5.3.1.

where $\sigma_{y}^{2}>0$ is the variance and $l_{\mathrm{c}}$ is the correlation length of $y$ that we set equal to $L / 8$. In the following, we use $\mu_{y}=-8.0179$ and $\sigma_{y}^{2}=0.3415$, values that were selected such that $K_{\mathrm{s}}$ has the same mean and variance than in the previous problems. Ordering the eigenvalues in decreasing order and truncating the KLE to the $N_{\mathrm{KL}}$ dominant modes, we obtain the following approximation

$$
K_{\mathrm{s}}(z, \theta) \simeq K_{\mathrm{s}}^{\mathrm{KL}}(z, \theta):=\exp \left[\mu_{y}+\sum_{i=1}^{N_{\mathrm{KL}}} \sqrt{\lambda_{i}} y_{i}(z) \eta_{i}(\theta)\right], \quad\left(\eta_{1}, \ldots, \eta_{N_{\mathrm{KL}}}\right) \sim \mathcal{N}(0, I) .
$$

In the remaining of the section we use $N_{\mathrm{KL}}=8$ leading to a truncation error on $y$ less than $1 \%$. We finally set $\xi_{i+2}:=\eta_{i}$ such that with the uncertain boundary and initial conditions, the pressure head $\psi_{h}(z, \xi)$ is parameterized with a total of $N_{\mathrm{KL}}+2=10$ independent random variables.

The constant (over the space domain) initial condition is not an equilibrium solution of the Richards' equation and the pressure head can lose monotonicity for non-homogeneous $K_{\mathrm{s}}$. An immediate way to recover monotonicity is to construct the PC-LP approximation of the hydraulic head, $\psi_{h}(z)-z$, which is almost surely monotonic and from which the pressure head can be easily recovered. However, the monotonicity w.r.t. $z$ of the QoI may be compromised by the PC expansion of the level points, in particular when many levels are considered, due to external aliasing. This problem is illustrated in Fig. 14. The plots in Fig. 14a and Fig. 14b compare for the same value of $\xi$ the PC-LP solution using a PSP grid with $\ell=3$ and 4 respectively, and the same number of levels $N_{1}=20$. The effect of the external aliasing can be seen with a spurious monotonicity loss for $\ell=4$ in the region with the highest gradient (see close-view sub-panels). 
Here, the complex interactions between the top boundary condition and the non-homogeneous $K_{\mathrm{s}}$ are responsible for the external aliasing, and a larger PSP level would be necessary to eventually properly capture high order interactions between the random inputs. To prove this point, we substitute the PSP method with a simple ordinary least squares (OLS) method, based on the same set of PSP nodes and PC basis, in the construction of the level points. As seen in the plot of Fig. 14c, the OLS approach is much less sensitive to external aliasing and presents a more satisfying approximation. It will be used in the remaining numerical experiments.

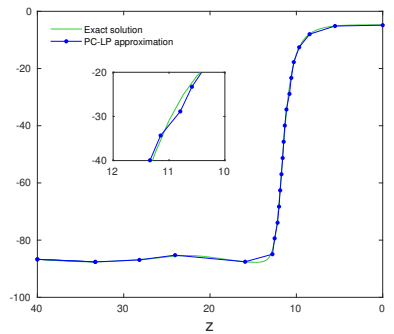

(a) PSP - level $\ell=3$

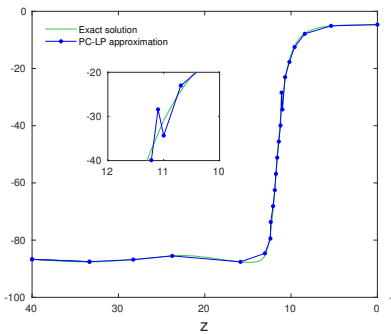

(b) PSP - level $\ell=4$

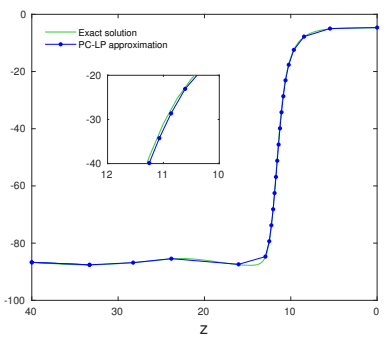

(c) OLS - level $\ell=4$

Figure 14: Illustration of the external aliasing in the PC-LP approximation with the PSP (left and center panels) and OLS (right panel) methods for $N_{\mathrm{l}}=20$. Case of non-homogeneous $K_{\mathrm{s}}$.

Fig. 15 presents the PC-LP approximations of the pressure head for $N_{1}=30$ and the OLS method based on the PSP nodes and basis at $\ell=4$. Compared are the approximations at the same value of $\boldsymbol{\xi}$ for a uniform (Fig. 15a) and adapted (Fig. 15b) discretizations of the range. We observe that the uniform discretization of the range provides a fairly faithful approximation, with sufficiently many level points in the bottom area $z>15$, owing to the representation of the hydraulic head. The largest error for the uniform discretization appears where the pressure head has the largest curvature (in space) and the adaptive method indeed succeeds in reducing the error in these areas, see Fig. $15 \mathrm{~b}$.

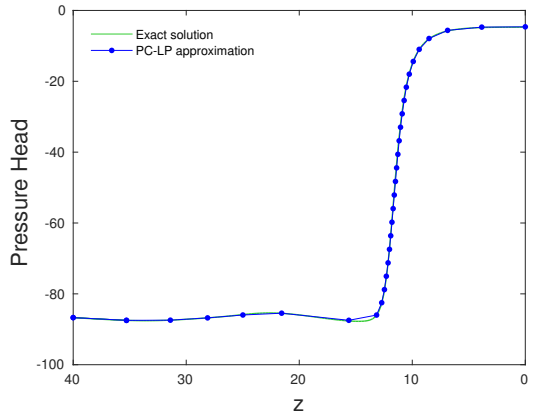

(a) Uniform discretization

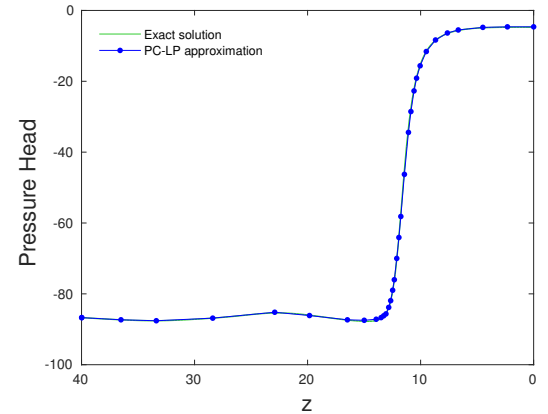

(b) Adapted discretization

Figure 15: PC-LP approximations for the uniform and adapted level selection. Numerical parameters are $N_{\mathrm{l}}=30$, OLS-level $\ell=4$. Case of non-homogeneous $K_{\mathrm{s}}$.

The improvement brought by the adaptive method remains however limited as it can be appreciated from Fig. 16 depicting the error $\widehat{e}(z)$ given by (23). The adaptive approach better balances the error level in the spatial domain but, contrary to the previous examples, the adaptivity does not bring significant error reduction. This is due to the dominant contribution of the high-range value for the hydraulic head at the top boundary, i.e $U_{+}(\xi)$ in the notations of Section 3, to the PC-LPM error. As $U_{+}(\boldsymbol{\xi})$ appears in the PC-LP approximation everywhere, see (13), its error is felt for all $x$, even though its effect decays with the local level value $v$. The adaptation cannot recover from this error which can be only reduced by increasing the quality of the PC approximation of $\mathcal{U}_{+}(\boldsymbol{\xi})$. As a result, for the range of numerical parameters tested, the adaptivity improvement on the spatially integrated error $\widehat{E}$ given by (24) is marginal, as shown in Table 2. In addition, the adaptivity improvement decays with $N_{1}$, as the PC error becomes more and more dominant. 


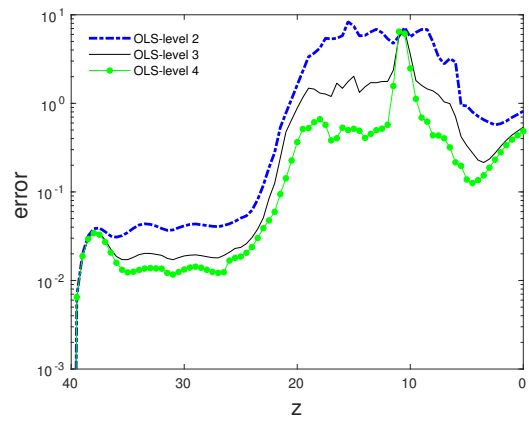

(a) Uniform discretization

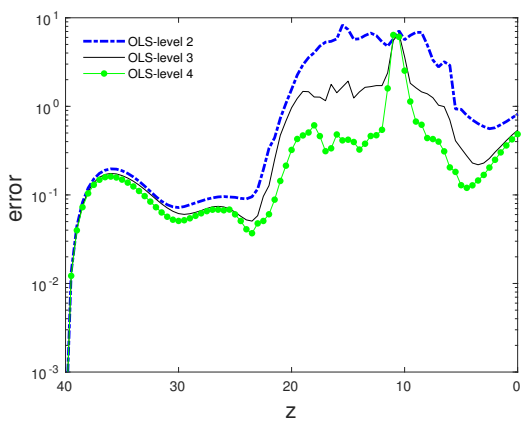

(b) Adapted discretization

Figure 16: Error $\widehat{e}(z)$ for the uniform and adapted level selection and $N_{1}=30$. The errors are reported for the OLS method on PSP nodes and basis of increasing levels $\ell=2,3$ and 4 . Case of non-homogeneous $K_{\mathrm{s}}$.

\begin{tabular}{|ccc|ccc|ccc|}
\hline & & & \multicolumn{3}{|c|}{ Uniform discretization } & \multicolumn{3}{c|}{ Adapted discretization } \\
$\ell$ & $N_{\mathrm{p}}$ & $|\mathcal{K}|$ & $N_{\mathrm{l}}=20$ & $N_{\mathrm{l}}=30$ & $N_{\mathrm{l}}=40$ & $N_{\mathrm{l}}=20$ & $N_{\mathrm{l}}=30$ & $N_{\mathrm{l}}=40$ \\
\hline 2 & 241 & 76 & 21.1 & 20.8 & 20.5 & 20.6 & 20.6 & 20.5 \\
3 & 2001 & 416 & 9.22 & 8.67 & 8.57 & 8.84 & 8.61 & 8.59 \\
4 & 13441 & 1966 & 7.28 & 6.93 & 6.89 & 7.00 & 6.86 & 6.83 \\
\hline
\end{tabular}

Table 2: Integrated error $\widehat{E}$ for number of levels $N_{\mathrm{l}}=20,30$ and 40. The errors are reported for the OLS method on PSP nodes and basis of increasing levels $\ell=2,3$ and 4 . The number $N_{\mathrm{p}}$ of PSP nodes and the dimension $|\mathcal{K}|$ of the PC bases are also indicated. Case of non-homogeneous $K_{\mathrm{s}}$.

\section{Conclusion}

We have proposed a novel method based on level points (LP) and Polynomial Chaos (PC) expansion to approximate one-dimensional uncertain fields. The construction involves a piecewise linear interpolation over a mesh corresponding to uncertain level points associated to selected level values. The resulting PC-LP method presents many advantages. First, it is less susceptible to Gibbs phenomenon, even for steep or discontinuous dependences, provided that level points are smoothly depending on the uncertain parameters. Second, for the examples considered in this work, the method was shown to be more accurate and computationally more efficient than a direct polynomial chaos approximation. In particular, low-order polynomial series can be used to approximate the stochastic LPs, requiring a significantly reduced computational effort to construct the approximation, compared to direct PC expansion which needs larger PC bases to yield similar accuracy. Also, the levels can be selected adaptively to minimize the interpolation error, with further improvement of the computational efficiency. From the implementation standpoint, the method is also attractive because the PC expansions of the LPs are non-intrusively computed; in fact, any non-intrusive strategy can be used. Finally, the computational overhead for the evaluation and sampling of the approximation is marginal unless the deterministic model is computationally cheap. Even when the adaptation of the levels is made for each sample, the overhead would remain negligible as the adapted construction proceeds with the same set of deterministic evaluations of the field, corresponding to the sparse grid nodes.

One essential limitation of the method is the requested (spatial) monotonicity of the field. This assumption can be alleviated in situations where the number of level points associated with a level value is not dependent on the uncertain parameters. Alternatively, one can handle non-monotonic situations by relying on (invertible) transformations of the original field to recover monotonicity, as it was done in the heterogeneous soil problem where the approximation of the hydraulic head (pressure head plus elevation head) has been considered instead of the pressure head. Another important limitation concerns the restriction of the method to one-dimensional spatial fields. The generalization of the level points to level curves and level surfaces appears as the main challenge to apply the method to fields in two and three space dimensions, respectively. For groundwater flow applications, we are currently extending the PC-LP method to 2 and 3 space dimensions, constraining the LPs to be along particular one-dimensional curves corresponding 
to the streamlines of a characteristic flow-field.

\section{References}

[1] Alexanderian, A., Le Maître, O., Najm, H., Iskandarani, M., Knio, O.: Multiscale stochastic preconditioners in non-intrusive spectral projection. SIAM J. Sci. Comp. 50(2), 306-340 (2012). DOI 10.1007/s10915-011-9486-2. URL http://dx. doi . org/10.1007/ S10915-011-9486-2

[2] Alexanderian, A., Rizzi, F., Rathinam, M., Le Maître, O., Knio, O.: Preconditioned bayesian regression for stochastic chemical kinetics. J. Sci. Comput. 58(3), 592-626 (2014). DOI 10.1007/s10915-013-9745-5. URL https://doi .org/10.1007/s10915-013-9745-5

[3] Brooks, R.H., Corey, A.T.: Hydraulic Properties of Porous Media. Colorado State University Hydrology Papers. Colorado State University (1964). URL http://books.google.com.pe/books?id=F \_1HOgAACAAJ

[4] Chantrasmi, T., Doostan, A., Iaccarino, G.: Padé-legendre approximants for uncertainty analysis with discontinuous response surfaces. J. Comput. Phys. 228(19), 7159 - 7180 (2009). DOI https://doi.org/10.1016/j.jcp.2009.06.024. URL http://www. sciencedirect. com/science/article/pii/s002199910900326X

[5] Conrad, P.R., Marzouk, Y.M.: Adaptive smolyak pseudospectral approximations. SIAM J. Sci. Comp. 35(6), A2643-A2670 (2013). DOI 10.1137/120890715. URL https: / / doi.org/10.1137/120890715

[6] Constantine, P.G., Eldred, M.S., Phipps, E.T.: Sparse pseudospectral approximation method. Comput. Methods Appl. Mech. Engrg. 229, $1-12(2012)$

[7] Ghanem, R.G., Spanos, S.D.: Stochastic Finite Elements: a Spectral Approach. Springer Verlag (1991)

[8] Karhunen, K.: Über lineare Methoden in der Wahrscheinlichkeitsrechnung. Annales Academiae scientiarum Fennicae: Mathematica Physica. Universitat Helsinki (1947). URL https : / / books. google.com/books? id=bGUUAQAAIAAJ

[9] Le Maître, O., Knio, O.M.: Spectral Methods for Uncertainty Quantification. Scientific Computation. Springer (2010)

[10] Le Maître, O., Mathelin, L., Knio, O.M., Hussaini, M.Y.: Asynchronous time integration for polynomial chaos expansion of uncertain periodic dynamics. Discret. Contin. Dyn. Sys. 28(1), 199-226 (2010). DOI 10.3934/dcds.2010.28.199. URL http://aimsciences . org/journals/displayArticlesnew.jsp?paperID=5069

[11] Le Maître, O., Najm, H., Ghanem, R., Knio, O.: Multi-resolution analysis of Wiener-type uncertainty propagation schemes. J. Comput. Phys. 197(2), 502-531 (2004). DOI 10.1016/j.jcp.2003.12.020. URL http://dx. doi.org/10.1016/j.jcp.2003.12.020

[12] Le Maître, O., Reagan, M.T., Najm, H.N., Ghanem, R.G., Knio, O.M.: A stochastic projection method for fluid flow ii.: Random process. J. Comput. Phys. 181(1), 9-44 (2002). DOI 10.1006/jcph.2002.7104. URL http://dx. doi.org/10.1006/jcph.2002.7104

[13] Poëtte, G., Lucor, D.: Non intrusive iterative stochastic spectral representation with application to compressible gas dynamics. J. Comput. Phys. 231(9), 3587-3609 (2012). DOI 10.1016/j.jcp.2011.12.038. URL http: / /dx.doi.org/10.1016/j.jcp.2011.12.038

[14] Quarteroni, A.M., Sacco, R., Saleri, F.: Numerical Mathematics. Texts in Applied Mathematics. Springer New York (2006). URL https : //books.google.com/books?id=FA3 \_DQAAQBAJ

[15] Reagan, M.T., Najm, H.M., Ghanem, R.G., Knio, O.M.: Uncertainty quantification in reacting-flow simulations through non-intrusive spectral projection. Combust. and Flame 132(3), 545 - 555 (2003). DOI https://doi.org/10.1016/S0010-2180(02)00503-5. URL http: //www . sciencedirect.com/science/article/pii/s0010218002005035

[16] Richards, L.: Capillary conduction of liquids through porous mediums. Physics 1, 318-333 (1931)

[17] Smolyak, S.: Quadrature and interpolation formulas for tensor products of certain classes of functions. Dokl. Akad. Nauk SSSR 4(240-243), 123 (1963)

[18] Sochala, P., Le Maître, O.: Polynomial Chaos expansion for subsurface flows with uncertain soil parameters. Adv. Water. Resour. 62, 139-154 (2013). DOI 10.1016/j.advwatres.2013.10.003. URL https : / / hal.archives-ouvertes.fr/hal-00931639

[19] Tryoen, J., Le Maître, O., Ern, A.: Adaptive anisotropic spectral stochastic methods for uncertain scalar conservation laws. SIAM J. Sci. Comp. 34(5), A2459-A2481 (2012). DOI 10.1137/120863927. URL https://doi .org/10.1137/120863927

[20] Tryoen, J., Le Maître, O., Ndjinga, M., Ern, A.: Intrusive Galerkin methods with upwinding for uncertain nonlinear hyperbolic systems. J. Comput. Phys. 229(18), 6485 - 6511 (2010). DOI https://doi.org/10.1016/j.jcp.2010.05.007. URL http: //www. sciencedirect. com/science/article/pii/s0021999110002688

[21] Wan, X., Karniadakis, G.: Multi-element generalized polynomial chaos for arbitrary probability measures. SIAM J. Sci. Comp. 28(3), 901-928 (2006)

[22] Winokur, J., Kim, D., Bisetti, F., Le Maître, O., Knio, O.M.: Sparse pseudo spectral projection methods with directional adaptation for uncertainty quantification. J. Sci. Comput. 68(2), 596-623 (2016). DOI 10.1007/s10915-015-0153-x. URL https://doi.org/10. $1007 /$ s10915-015-0153-x

[23] Xiu, D., Karniadakis, G.: Supersensitivity due to uncertain boundary conditions. Int. J. Numer. Meth. Engng. 61(12), 2114-2138 (2004) 